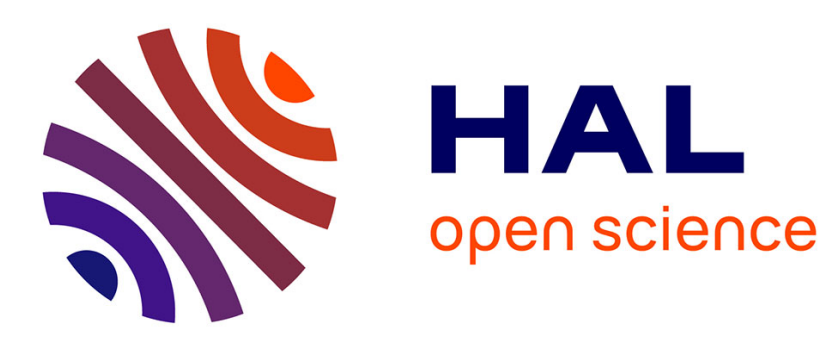

\title{
NO3 full-dimensional potential energy surfaces and ground state vibrational levels revisited
}

\author{
Alexandra Viel, Wolfgang Eisfeld
}

\section{To cite this version:}

Alexandra Viel, Wolfgang Eisfeld. NO3 full-dimensional potential energy surfaces and ground state vibrational levels revisited. Chemical Physics, 2018, 509, pp.81-90. 10.1016/j.chemphys.2018.01.003 . hal-01725478

\section{HAL Id: hal-01725478 \\ https://hal.science/hal-01725478}

Submitted on 8 May 2020

HAL is a multi-disciplinary open access archive for the deposit and dissemination of scientific research documents, whether they are published or not. The documents may come from teaching and research institutions in France or abroad, or from public or private research centers.
L'archive ouverte pluridisciplinaire HAL, est destinée au dépôt et à la diffusion de documents scientifiques de niveau recherche, publiés ou non, émanant des établissements d'enseignement et de recherche français ou étrangers, des laboratoires publics ou privés. 


\title{
$\mathrm{NO}_{3}$ full-dimensional potential energy surfaces and ground state vibrational levels revisited
}

\author{
Alexandra Viel \\ Univ Rennes, CNRS, IPR (Institut de Physique de Rennes) - UMR 6251, F-35000 Rennes, France \\ Wolfgang Eisfeld \\ Theoretische Chemie, Universität Bielefeld, Postfach 100131, D-33501 Bielefeld, Germany
}

\begin{abstract}
A new full-dimensional (6D) diabatic potential energy surface (PES) model is presented representing the five lowest PESs corresponding to the $\tilde{X}^{2} A_{2}^{\prime}, \tilde{A}^{2} E^{\prime \prime}$, and $\tilde{B}^{2} E^{\prime}$ electronic states of the nitrate radical $\left(\mathrm{NO}_{3}\right)$. It is based on high-level $a b$ initio calculations of roughly 90000 energy data over a wide range of nuclear configurations and represents the energies with a root mean-squares (rms) error of about $100 \mathrm{~cm}^{-1}$. An accurate dipole surface was developed for the $\tilde{X}$ state as well. The new PES model is used to re-investigate the infra-red (IR) spectrum corresponding to the electronic ground state by full quantum dynamics simulations. Vibrational eigenstates, IR transition probabilities, and isotopic shifts are computed and analyzed. Levels up to $2000 \mathrm{~cm}^{-1}$ are obtained and show good to excellent agreement with known experimental values. Some larger deviations are observed and discussed as well. The new results are in agreement with previous theoretical studies that the disputed $\nu_{3}$ fundamental corresponds to a frequency of roughly $1022 \mathrm{~cm}^{-1}$ and that the prominent experimental feature observed at $1492 \mathrm{~cm}^{-1}$ is due to the $3^{1} 4^{1}\left(e^{\prime}\right)$ combination mode. Observed discrepancies in the IR intensities may be explained by coupling to the $\tilde{B}$ state which is also analysed by diabatic decomposition of the eigenstates.
\end{abstract}

\section{Introduction}

$\mathrm{NO}_{3}$, one of the first known free radicals, is the subject of intense research and lively controversy still more than 130 years after its first discovery[1] and almost 90 years after its first spectroscopic characterization[2]. A number of idiosyncrasies have sparked the interest of experimentalists and theoreticians alike - besides its high relevance in atmospheric chemistry. [3, 4] Three electronic states are known to date, the ${ }^{2} A_{2}^{\prime}$ ground state, the first excited state of ${ }^{2} E^{\prime \prime}$ symmetry, and the second excited state of ${ }^{2} E^{\prime}$ symmetry. The $\tilde{B}$ state absorbs strongly in the visible and has been measured as early as in the 1930s. [2, 5, 6] This state is subject to Eisfeld)

Email addresses: alexandra.viel@univ-rennes1.fr (Alexandra Viel), wolfgang.eisfeld@uni-bielefeld.de (Wolfgang 
photodissociation though it shows a long life-time at the same time.[7, 8, 9, 10, 11] The $\tilde{A}$ state is difficult to observe in direct absorption because transitions are formally dipole-forbidden. However, it has been observed first by photodetachment spectroscopy from the anion.[12] Much later it was measured by cavity ring-down spectroscopy in a number of studies.[13, 14, 15, 16] This state also has been observed in neon matrices at 4.3 K.[17] The analysis of these spectra is still under way. Finally, the $\tilde{X}$ state can be readily studied by a number of high-resolution spectroscopic techniques. The vibrational and ro-vibrational levels have been studied by several groups with different techniques.[18, 19, 20, 21, 22, 23, 24, 25, 26, 27, 28, 29, 30, 31, 32, 33] A lively debate has developed about the assignment of a feature around $1492 \mathrm{~cm}^{-1}$ caused by a theoretical study[34] proposing that this level may not be the $\nu_{3}$ fundamental but a $1^{1} 4^{1}$ combination band. In a follow-up study, this theoretical assignment was corrected to $3^{1} 4^{1}[57]$.

The theoretical treatment of $\mathrm{NO}_{3}$ has been no less controversial due to intrinsic problems of the electronic structure. There has been a long debate about the equilibrium geometry, $C_{2 v}$ vs. $D_{3 h},[35,36,37,38,39$, $40,41,42,43,44,45]$ and finally it could be shown that the distorted $C_{2 v}$ geometries are due to artificial symmetry-breaking of the electronic wave function.[46] A comprehensive discussion of this can be found in ref. [46, 47]. A further controversy was the question whether or not a stable isomer ONOO (peroxy nitrate) exists. Its existence at least as intermediate has been proposed by several groups based on experiment and theory.[48, 49, 50, 51, 40] The experimental evidence turned out to be due to systematic errors.[52] Finally, we could show that the theoretical support for a stable ONOO isomer was also due to inappropriate use of single-reference electronic structure methods. [53] Several quantum dynamics studies also appeared treating various spectroscopic aspects. $[54,14,34,55,56,57,58,59,60,61,62]$ All except one of these studies utilize a vibronic coupling or diabatic representation of the electronic Hamiltonian to obtain an analytical potential energy surface (PES) model. We also present an accurate diabatic 5-state PES model in the present study which is an extension of our previous work. [61, 62]

The reason for using such a diabatic representation even for the ground state is the extreme anharmonicity in the corresponding PES which is due to strong couplings to the Jahn-Teller (JT) active excited $\tilde{B}$ state. The presence of conical intersections and the resulting breakdown of the Born-Oppenheimer (BO) approximation for the excited states renders such an approach very desirable.[63] The nuclear and electronic degrees of freedom are coupled in the excited states, which has to be accounted for in any reasonable theoretical treatment. By contrast, the electronic ground state could be treated adiabatically without any problems but the adiabatic PES is difficult to be fitted. This turns out to be much easier in the fully coupled diabatic representation.

The benefits of a diabatic or rather quasi-diabatic representation of the electronic Hamiltonian and corresponding PES matrix are well-known by now.[63, 64, 65, 66, 67, 68, 69, 70, 71, 72, 73, 74] Though the linear vibronic coupling method has been tremendously successful,[75] it is also too limited to yield accurate PESs. For this reason we and others have been developing methods to diabatize electronic structure 
data $[76,77,78,79,80,81,82,83,84,85,86,87,88,89,90]$ and to generate and represent coupled PESs that are accurate over a large range of nuclear configurations. [91, 92, 93, 94, 95, 96, 97, 98, 99, 100, 101, 102, $103,104,105,106,107,108]$ We recently applied our methodology to the development of a highly accurate diabatic representation of the ${ }^{2} E^{\prime \prime}$ first excited electronic state of $\mathrm{NO}_{3}$, which is a prototypical Jahn-Teller system in which the proper treatment of the direct dissociation asymptotes is very important.[61] In the present study, we extend this PES model for a detailed analysis of the nuclear dynamics in the $\tilde{X}^{2} A_{2}^{\prime}$ state.

The paper is organized as follows. Section 2 provides a brief description of the diabatic PES model and the dipole surface model. Section 3 provides the technical and numerical details of the vibrational level computations. Results of the numerical simulations are given, analyzed, and discussed in detail in Section 4 and the conclusions of this work are summarized in Section 5 .

\section{Diabatic potential model}

The electronic ground state $\tilde{X}^{2} A_{2}^{\prime}$ is non-degenerate and could be represented by a single adiabatic PES in principle. In fact, this has been done.[60] However, the $\tilde{X}$ state is coupled to the Jahn-Teller active $\tilde{B}$ state of ${ }^{2} E^{\prime}$ symmetry by a significantly strong pseudo-JT coupling as was first stated by Mayer et al.[54] and confirmed much later by Stanton.[34] This coupling has an effect on the shape of the ground state PES making it harder to represent the data as a single uncoupled PES. Furthermore, we are interested in the photo chemistry and absorption spectroscopy of $\mathrm{NO}_{3}$ and both low-lying excited states, the $\tilde{A}^{2} E^{\prime \prime}$ as well as the $\tilde{B}^{2} E^{\prime}$ state, are JT active with a conical intersection at $D_{3 h} / C_{3 v}$ geometries. It is of great advantage to represent such a system in the diabatic electronic basis. Our aim is to develop a full-dimensional, global, and accurate diabatic model for all relevant electronic states of this complicated system. So far the diabatic treatment of $\mathrm{NO}_{3}$ was mostly restricted to the simple linear and quadratic vibronic coupling approach.[54, 34, 58] We extended this treatment to higher order JT couplings[100, 101] and global PESs step by step first for the isolated ${ }^{2} E^{\prime \prime}$ state and used that system for the development of our methodology.[55, 56, 61]

Here we extend our approach to the diabatic representation of the three lowest electronic states of $\mathrm{NO}_{3}$ including five state components and thus yielding a $5 \times 5$ diabatic PES matrix depending on the six nuclear coordinates $Q_{i}$. The diabatic electronic basis states are used in the order $\left|{ }^{2} A_{2}^{\prime}\right\rangle,\left|{ }^{2} E_{x}^{\prime \prime}\right\rangle,\left|{ }^{2} E_{y}^{\prime \prime}\right\rangle,\left|{ }^{2} E_{x}^{\prime}\right\rangle$, and $\left|{ }^{2} E_{y}^{\prime}\right\rangle$ in the following. This yields the diabatic matrix

$$
\boldsymbol{E}^{d}(\boldsymbol{Q})=\left(\begin{array}{ccccc}
\mathcal{V}_{\tilde{X}}(\boldsymbol{Q}) & 0 & 0 & 0 & 0 \\
0 & \mathcal{V}_{\tilde{A}}(\boldsymbol{Q}) & 0 & 0 & 0 \\
0 & 0 & \mathcal{V}_{\tilde{A}}(\boldsymbol{Q}) & 0 & 0 \\
0 & 0 & 0 & \mathcal{V}_{\tilde{B}}(\boldsymbol{Q}) & 0 \\
0 & 0 & 0 & 0 & \mathcal{V}_{\tilde{B}}(\boldsymbol{Q})
\end{array}\right)
$$




$$
\begin{aligned}
& +\left(\begin{array}{ccccc}
0 & 0 & 0 & 0 & 0 \\
0 & \mathcal{W}_{\tilde{A}}(\boldsymbol{Q}) & \mathcal{Z}_{\tilde{A}}(\boldsymbol{Q}) & 0 & 0 \\
0 & \mathcal{Z}_{\tilde{A}}(\boldsymbol{Q}) & -\mathcal{W}_{\tilde{A}}(\boldsymbol{Q}) & 0 & 0 \\
0 & 0 & 0 & \mathcal{W}_{\tilde{B}}(\boldsymbol{Q}) & \mathcal{Z}_{\tilde{B}}(\boldsymbol{Q}) \\
0 & 0 & 0 & \mathcal{Z}_{\tilde{B}}(\boldsymbol{Q}) & -\mathcal{W}_{\tilde{B}}(\boldsymbol{Q})
\end{array}\right) \\
& +\left(\begin{array}{ccccc}
0 & \mathcal{W}_{\tilde{X} \tilde{A}}(\boldsymbol{Q}) & -\mathcal{Z}_{\tilde{X} \tilde{A}}(\boldsymbol{Q}) & \mathcal{W}_{\tilde{X} \tilde{B}}(\boldsymbol{Q}) & -\mathcal{Z}_{\tilde{X} \tilde{B}}(\boldsymbol{Q}) \\
\mathcal{W}_{\tilde{X} \tilde{A}}(\boldsymbol{Q}) & 0 & 0 & \mathcal{V}_{\tilde{A} \tilde{B}}(\boldsymbol{Q})+\mathcal{W}_{\tilde{A} \tilde{B}}(\boldsymbol{Q}) & \mathcal{Z}_{\tilde{A} \tilde{B}}(\boldsymbol{Q}) \\
-\mathcal{Z}_{\tilde{X} \tilde{A}}(\boldsymbol{Q}) & 0 & 0 & \mathcal{Z}_{\tilde{A} \tilde{B}}(\boldsymbol{Q}) & \mathcal{V}_{\tilde{A} \tilde{B}}(\boldsymbol{Q})-\mathcal{W}_{\tilde{A} \tilde{B}}(\boldsymbol{Q}) \\
\mathcal{W}_{\tilde{X} \tilde{B}}(\boldsymbol{Q}) & \mathcal{V}_{\tilde{A} \tilde{B}}(\boldsymbol{Q})+\mathcal{W}_{\tilde{A} \tilde{B}}(\boldsymbol{Q}) & \mathcal{Z}_{\tilde{A} \tilde{B}}(\boldsymbol{Q}) & 0 & 0 \\
-\mathcal{Z}_{\tilde{X} \tilde{B}}(\boldsymbol{Q}) & \mathcal{Z}_{\tilde{A} \tilde{B}}(\boldsymbol{Q}) & \mathcal{V}_{\tilde{A} \tilde{B}}(\boldsymbol{Q})-\mathcal{W}_{\tilde{A} \tilde{B}}(\boldsymbol{Q}) & 0 & 0
\end{array}\right)
\end{aligned}
$$

in which the functions $\mathcal{V}, \mathcal{W}$, and $\mathcal{Z}$ are symmetry-adapted power expansions in terms of symmetry-adapted nuclear coordinates. The corresponding expansion coefficients are determined by the diabatization approach utilizing computed electronic structure data (see below). The basic symmetry monomials have been derived by us and others before and need not to be repeated here.[100, 101, 61] For the present work we included all terms up to fourth order including all possible multi-mode couplings. Special attention has to be paid to the symmetry-breaking umbrella coordinate $Q_{2}$ which transforms as $a_{2}^{\prime \prime}$. Only monomials with odd powers $Q_{2}^{2 n+1}$ are allowed in the coupling functions $\mathcal{W}_{\tilde{X} \tilde{A}}, \mathcal{Z}_{\tilde{X} \tilde{A}}, \mathcal{V}_{\tilde{A} \tilde{B}}, \mathcal{W}_{\tilde{A} \tilde{B}}$, and $\mathcal{Z}_{\tilde{A} \tilde{B}}$. All other functions must only contain monomials with even powers $Q_{2}^{2 n}$ of this coordinate.

The coordinates are constructed from a set of primitive valence coordinates. These are the three $\mathrm{N}-\mathrm{O}$ distances, an umbrella angle $\theta$ defined as the angle of any of the $\mathrm{N}-\mathrm{O}$ bonds to the plane normal to the trisector, which is a line in space going through the central $\mathrm{N}$ atom to which all three $\mathrm{N}-\mathrm{O}$ bonds form the same angle, and the three $\mathrm{O}-\mathrm{N}-\mathrm{O}$ angles obtained from projection of the $\mathrm{N}-\mathrm{O}$ bonds onto the normal plane corresponding to the trisector.[61] Then the three $\mathrm{N}-\mathrm{O}$ distances are transformed first into displacements $\Delta r_{i}$ with respect to the equilibrium distance $r_{e}$ of the ground state and then into Morse coordinates

$$
m_{i}\left(\Delta r_{i}\right)=1-e^{-\alpha \Delta r_{i}}, \quad i=1,2,3 .
$$

These Morse coordinates are symmetry-adapted by the well known point-group Clebsch-Gordan coefficients for $D_{3 h}$ to yield the $a_{1}^{\prime}$ totally symmetric stretch and a set of $e^{\prime}$ asymmetric stretch coordinates:

$$
\left(\begin{array}{l}
Q_{1} \\
Q_{3} \\
Q_{4}
\end{array}\right)=\left(\begin{array}{ccc}
\frac{1}{\sqrt{3}} & \frac{1}{\sqrt{3}} & \frac{1}{\sqrt{3}} \\
\frac{2}{\sqrt{6}} & -\frac{1}{\sqrt{6}} & -\frac{1}{\sqrt{6}} \\
0 & \frac{1}{\sqrt{2}} & -\frac{1}{\sqrt{2}}
\end{array}\right)\left(\begin{array}{l}
m_{1} \\
m_{2} \\
m_{3}
\end{array}\right) .
$$

The three projected $\mathrm{O}-\mathrm{N}-\mathrm{O}$ angles are first divided by the product of the two $\mathrm{N}-\mathrm{O}$ distances forming the scaled angles

$$
\tilde{\alpha}_{i}=\frac{\alpha_{i}}{r_{j} r_{k}}, \quad i \neq j \neq k \in\{1,2,3\}
$$


and then symmetry-adapted exactly like the distances

$$
\left(\begin{array}{c}
Q_{5} \\
Q_{6}
\end{array}\right)=\left(\begin{array}{ccc}
\frac{2}{\sqrt{6}} & -\frac{1}{\sqrt{6}} & -\frac{1}{\sqrt{6}} \\
0 & \frac{1}{\sqrt{2}} & -\frac{1}{\sqrt{2}}
\end{array}\right)\left(\begin{array}{c}
\alpha_{1} \\
\alpha_{2}
\end{array}\right) .
$$

The totally symmetric linear combination is discarded because it is an inappropriate coordinate. The above defined umbrella angle is used instead, which is scaled by the $\mathrm{N}-\mathrm{O}$ distances as

$$
Q_{2}=\theta \frac{r_{e}^{3}}{r_{1} r_{2} r_{3}}
$$

To give an idea how the diabatic PES functions of eq. (1) actually look like, we present explicitly the two dynamic pseudo-JT coupling elements $\mathcal{W}_{\tilde{X} \tilde{A}}$ and $\mathcal{Z}_{\tilde{X} \tilde{A}}$ in terms of the above coordinates, which read

$$
\begin{aligned}
\mathcal{W}_{\tilde{X} \tilde{A}}(\boldsymbol{Q})= & p_{1}^{(1)} Q_{2} \\
& +p_{1}^{(2)} Q_{2} Q_{3}+p_{2}^{(2)} Q_{2} Q_{5}+p_{3}^{(2)} Q_{1} Q_{2} \\
& +p_{1}^{(3)} Q_{2}^{3}+p_{2}^{(3)} Q_{2}\left(Q_{3}^{2}-Q_{4}^{2}\right)+p_{3}^{(3)} Q_{2}\left(Q_{5}^{2}-Q_{6}^{2}\right)+p_{4}^{(3)} Q_{2}\left(Q_{3} Q_{5}-Q_{4} Q_{6}\right) \\
& +p_{5}^{(3)} Q_{1} Q_{2} Q_{3}+p_{6}^{(3)} Q_{1} Q_{2} Q_{5} \\
& +p_{1}^{(4)} Q_{2}\left(Q_{3}^{3}+Q_{3} Q_{4}^{2}\right)+p_{2}^{(4)} Q_{2}\left(Q_{5}^{3}+Q_{5} Q_{6}^{2}\right)+p_{3}^{(4)} Q_{2}\left(Q_{3}^{2} Q_{5}+Q_{4}^{2} Q_{5}\right) \\
& +p_{4}^{(4)} Q_{2}\left(Q_{3} Q_{6}^{2}+Q_{3} Q_{5}^{2}\right)+p_{5}^{(4)} Q_{2}\left(2 Q_{3} Q_{4} Q_{6}+Q_{3}^{2} Q_{5}-Q_{4}^{2} Q_{5}\right) \\
& +p_{6}^{(4)} Q_{2}\left(Q_{3} Q_{5}^{2}+2 Q_{4} Q_{5} Q_{6}-Q_{3} Q_{6}^{2}\right)+p_{7}^{(4)} Q_{1} Q_{2}\left(Q_{3}^{2}-Q_{4}^{2}\right)+p_{8}^{(4)} Q_{1} Q_{2}\left(Q_{5}^{2}-Q_{6}^{2}\right) \\
& +p_{9}^{(4)} Q_{1} Q_{2}\left(Q_{3} Q_{5}-Q_{4} Q_{6}\right)+p_{10}^{(4)} Q_{1}^{2} Q_{2} Q_{3}+p_{11}^{(4)} Q_{1}^{2} Q_{2} Q_{5}+p_{12}^{(4)} Q_{1} Q_{2}^{3} \\
& +p_{13}^{(4)} Q_{2}^{3} Q_{3}+p_{14}^{(4)} Q_{2}^{3} Q_{5}
\end{aligned}
$$

and

$$
\begin{aligned}
\mathcal{Z}_{\tilde{X} \tilde{A}}(\boldsymbol{Q})= & p_{1}^{(1)} Q_{2} \\
& +p_{1}^{(2)} Q_{2} Q_{4}+p_{2}^{(2)} Q_{2} Q_{6}+p_{3}^{(2)} Q_{1} Q_{2} \\
& +p_{1}^{(3)} Q_{2}^{3}-2 p_{2}^{(3)} Q_{2} Q_{3} Q_{4}-2 p_{3}^{(3)} Q_{2} Q_{5} Q_{6}-p_{4}^{(3)} Q_{2}\left(Q_{3} Q_{6}+Q_{4} Q_{5}\right) \\
& +p_{5}^{(3)} Q_{1} Q_{2} Q_{4}+p_{6}^{(3)} Q_{1} Q_{2} Q_{6} \\
& +p_{1}^{(4)} Q_{2}\left(Q_{3}^{2} Q_{4}+Q_{4}^{3}\right)+p_{2}^{(4)} Q_{2}\left(Q_{5}^{2} Q_{6}+Q_{6}^{3}\right)+p_{3}^{(4)} Q_{2}\left(Q_{4}^{2} Q_{6}+Q_{3}^{2} Q_{6}\right) \\
& +p_{4}^{(4)} Q_{2}\left(Q_{4} Q_{6}^{2}+Q_{4} Q_{5}^{2}\right)+p_{5}^{(4)} Q_{2}\left(Q_{4}^{2} Q_{6}+2 Q_{3} Q_{4} Q_{5}-Q_{3}^{2} Q_{6}\right) \\
& +p_{6}^{(4)} Q_{2}\left(Q_{4} Q_{6}^{2}+2 Q_{3} Q_{5} Q_{6}-Q_{4} Q_{5}^{2}\right)-p_{7}^{(4)} 2 Q_{1} Q_{2} Q_{3} Q_{4}-p_{8}^{(4)} Q_{1} Q_{2} Q_{5} Q_{6} \\
& -p_{9}^{(4)} Q_{1} Q_{2}\left(Q_{3} Q_{6}+Q_{4} Q_{5}\right)+p_{10}^{(4)} Q_{1}^{2} Q_{2} Q_{4}+p_{11}^{(4)} Q_{1}^{2} Q_{2} Q_{6}+p_{12}^{(4)} Q_{1} Q_{2}^{3} \\
& +p_{13}^{(4)} Q_{2}^{3} Q_{4}+p_{14}^{(4)} Q_{2}^{3} Q_{6} .
\end{aligned}
$$

Note that both functions must share a common set of expansion parameters $p_{j}^{(n)}$ because these functions are strictly related by symmetry. The free parameters $p$ in the diabatic functions need to be determined based 
on accurate electronic structure calculations. The required energy data was computed at multi-configuration reference singles and doubles configuration interaction (MR-SDCI) level of theory using a modified triple- $\zeta$ basis. The technical details of the $a b$ initio calculations can be found in refs. [46, 47] These calculations have been performed in two different sets, a large 5D set for planar geometries and a smaller $6 \mathrm{D}$ one including displacement of the umbrella coordinate. In order to detect potential convergence issues of the ab initio data, these calculations are performed along well-defined straight random cuts through the PESs as described in refs. [109, 61, 90] The free parameters are then obtained by a non-linear least squares fitting algorithm based on the Marquardt-Levenberg method incorporated into a genetic algorithm. The diabatization is achieved by the requirement that the eigenvalues of the diabatic model need to optimally reproduce the adiabatic energies for all nuclear configurations in the data set. The fit is first performed for the $5 \mathrm{D}$ data restricted to planar nuclear configurations. This first fitting step is based on 86661 adiabatic energy data from the MRCI $a b$ initio calculations. The obtained parameters are then frozen and a second fit with a set of full $6 \mathrm{D}$ data (3 034 energies) is performed only optimizing the parameters for terms including displacements in the umbrella coordinate. An exponential weighting of the data is applied with respect to the reference energies at the ground state equilibrium geometry in order to improve the representation of the most relevant regions of the PESs which are of lower energy. This approach yields an unweighted root mean-squares (rms) error of below $100 \mathrm{~cm}^{-1}$ for all energies up to $1 \mathrm{eV}$ above the origin of each of the electronic states. The weighted rms error for all energies included into the fit spanning a range of several $\mathrm{eV}$ is only about $120 \mathrm{~cm}^{-1}$, demonstrating the excellent representation of the electronic structure data over a large range of nuclear configurations.

Finally, we also need the dipole surfaces in order to compute IR intensities. For simplicity this is only done in the adiabatic approximation for the electronic ground state. It turns out that the widely applied linear approximation is insufficient in the case of the $\mathrm{NO}_{3}$ ground state since strong multi-mode effects and non-linearity are present. A symmetry analysis of the three vector components of the dipole function $\vec{\mu}(\boldsymbol{Q})$ yields that it is properly represented in terms of the symmetry-adapted polynomials as

$$
\vec{\mu}(\boldsymbol{Q})=\left(\begin{array}{c}
\mathcal{W}_{\mu}(\boldsymbol{Q}) \\
\mathcal{Z}_{\mu}(\boldsymbol{Q}) \\
Q_{2}^{2 n+1} \mathcal{V}_{\mu}(\boldsymbol{Q})
\end{array}\right)
$$

We find that $\mu_{z}$ hardly depends on any other coordinates except $Q_{2}$ and thus it is simply modeled by the linear and cubic term in $Q_{2}$. The other two components $\mu_{x}$ and $\mu_{y}$ show strong multi-mode effects and are expanded to full third order including all multi-mode terms in the $\mathcal{Z}$ and $\mathcal{W}$ functions. The 14 free parameters are fitted with respect to a total of 612 ab initio data in full 6D and a satisfactory representation of the data was achieved by the above model. 


\section{Computational Details}

For the determination of the vibrational levels supported by the electronic ground state, we employed the MCTDH approach, $[110,111,112]$ which is a powerful scheme to describe wave functions of large systems. ${ }^{14} \mathrm{~N}^{16} \mathrm{O}_{3}$ as well as two other isotopologues have been considered. The state average and block diagonalization scheme as described in ref. [113] is used to compute vibrational levels. In our previous work on the first excited state of $\mathrm{NO}_{3}$ [62], we have shown that the six internal curvilinear coordinates as proposed in ref. [114] are convenient coordinates to describe $\mathrm{NO}_{3}$. These six internal curvilinear coordinates $\rho^{(\mathrm{cu})}, \vartheta^{(\mathrm{cu})}, \varphi^{(\mathrm{cu})}, \theta^{(\mathrm{cu})}, \phi^{(\mathrm{cu})}, \chi^{(\mathrm{cu})}$ are based on the three Radau vectors of the $\mathrm{AB}_{3}$ system. Planarity is given for $\theta^{(\mathrm{cu})}=\pi / 2$ where $\theta^{(\mathrm{cu})}$ is defined as the angle between each of the Radau vectors and the trisector of the Radau vectors. The exact kinetic energy operator cannot be used directly in the MCTDH implementation due to the fact that one of the terms cannot be expressed as sum of products of single coordinate terms. As proposed in ref. [114], the fourth order Taylor expansion is used here. This approximation has been tested on the computation of $\mathrm{NO}_{3}^{-}$vibrational and $\mathrm{NO}_{3}$ vibronic energy levels [62] for which we tested an alternative set of coordinates, namely the stereographic coordinates as defined in ref. [115]. The differences are below $1 \mathrm{~cm}^{-1}$.

These coordinates have indeed two qualities when studying $\mathrm{NO}_{3}$. First, the quite physical nature of these coordinates ensures that for the eigenvector representations the correlation between the coordinates is moderate. The consequence is a faster convergence of the MCTDH scheme with respect to the number of single particle functions. Second and most importantly, when using this particular set of coordinates one can easily evaluate the effect of the symmetry operators of the $C_{2 v}$ subgroup of the relevant $D_{3 h}$ symmetry group. With the $x y$ plane being the molecular plane, the $\sigma_{x y}$ reflection acts on the $\theta^{(\mathrm{cu})}$ only around the $\pi / 2$ symmetry point, the $\sigma_{x z}$ reflection acts on both $\varphi^{(\mathrm{cu})}$ and $\chi^{(\mathrm{cu})}$ around the $\pi / 4$ and the $\pi$ symmetry point, respectively, and the $C_{2}$ rotation acts on the three $\theta^{(\mathrm{cu})}, \varphi^{(\mathrm{cu})}$ and $\chi^{(\mathrm{cu})}$ angles. The correlation between $\mathrm{D}_{3 h}$ and $\mathrm{C}_{2 v}$ irreducible representations and corresponding characters of the two $\mathrm{C}_{2 v}$ reflections are given in Tab. 1.

Table 1: Relation between irreps in $C_{2 v}$ and $D_{3 h}$ within the orientational convention of this work. The characters with respect to the two $\sigma_{v}$ reflection of $C_{2 v}$ are also provided for convenience.

\begin{tabular}{crrr}
$\Gamma_{D_{3 h}}$, & $\Gamma_{C_{2 v}}$ & $\sigma_{v}(x y)$ & $\sigma_{v}(x z)$ \\
\hline$a_{1}^{\prime}$ or $e^{\prime}$ & $a_{1}$ & 1 & 1 \\
$a_{2}^{\prime}$ or $e^{\prime}$ & $b_{1}$ & 1 & -1 \\
$a_{1}^{\prime \prime}$ or $e^{\prime \prime}$ & $a_{2}$ & -1 & -1 \\
$a_{2}^{\prime \prime}$ or $e^{\prime \prime}$ & $b_{2}$ & -1 & 1 \\
\hline
\end{tabular}

Although the kinetic energy terms fulfill the sum of products of single particle constraint of operators to render the MCTDH approach most efficient, the potential term does not respect this requirement. Therefore, 
when studying the vibrational levels supported by the adiabatic ground state, the evaluation of the singlestate (or single-valued) potential term is carried out using the original CDVR scheme.[116] For the diabatic calculations employing the full $5 \times 5$ matrix operator the multi-state CDVR scheme is utilized.[102] Table 2 gives the basis set details, namely box range, underlying grid size $N$, and number of single particle functions $n$ which have been converged in order to ensure numerical accuracy of the final results to better than one $\mathrm{cm}^{-1}$.

Table 2: Wave function representations given by the number of single particle functions $(n)$, the number of Fourier points $(N)$ and the range of the underlying box [in a.u.] for the six curvilinear coordinates of hyperspherical type constructed using mass weighted Cartesian coordinates for ${ }^{14} \mathrm{~N}^{16} \mathrm{O}_{3},{ }^{15} \mathrm{NO}_{3}$ and ${ }^{14} \mathrm{~N}^{18} \mathrm{O}^{16} \mathrm{O}_{2}$.

\begin{tabular}{c|ccc|ccc|ccc} 
& \multicolumn{4}{|c|}{${ }^{14} \mathrm{~N}^{16} \mathrm{O}_{3}$} & \multicolumn{4}{c}{${ }^{15} \mathrm{~N}^{16} \mathrm{O}_{3}$} & \multicolumn{3}{c}{${ }^{14} \mathrm{~N}^{18} \mathrm{O}^{16} \mathrm{O}_{3}$} \\
\hline coord. & $n$ & $N$ & range & $n$ & $N$ & range & $n$ & $N$ & range \\
\hline$\rho^{(\mathrm{cu})}$ & 5 & 32 & {$[648: 753]$} & 5 & 32 & {$[648: 753]$} & 5 & 32 & {$[645: 765]$} \\
$\vartheta^{(\mathrm{cu})}$ & 7 & 32 & {$[0.855: 1.055]$} & 7 & 32 & {$[0.855: 1.055]$} & 7 & 32 & {$[0.855: 1.055]$} \\
$\varphi^{(\mathrm{cu})}$ & 7 & 32 & {$[0.685: 0.885]$} & 7 & 32 & {$[0.685: 0.885]$} & 7 & 32 & {$[0.685: 0.885]$} \\
$\theta^{(\mathrm{cu})}$ & 5 & 32 & {$[1.471: 1.671]$} & 5 & 32 & {$[1.471: 1.671]$} & 5 & 32 & {$[1.471: 1.671]$} \\
$\phi^{(\mathrm{cu})}$ & 9 & 32 & {$[0.827: 1.217]$} & 9 & 32 & {$[0.827: 1.217]$} & 9 & 32 & {$[0.807: 1.217]$} \\
$\chi^{(\mathrm{cu})}$ & 10 & 32 & {$[2.741: 3.541]$} & 10 & 32 & {$[2.741: 3.541]$} & 10 & 32 & {$[2.741: 3.541]$}
\end{tabular}

The vibrational energy levels are also computed in a complimentary way. A time-independent Hermite discrete variable representation (DVR) approach is used[117] and the corresponding Hamiltonian is diagonalized by an exact short iterative Lanczos method. Normal coordinates from an MRCI frequency calculation are used and vibrational angular momenta are ignored in the kinetic energy operator. Standard harmonic oscillator functions are chosen for the finite basis representation (FBR) and the kinetic energy is transformed into the corresponding DVR grid point basis. The six coordinates and their irreps in $D_{3 h}$ correspond to totally symmetric stretch $\left(\nu_{1}, \mathrm{a}_{1}^{\prime}\right)$, out-of-plane or umbrella bending $\left(\nu_{2}, \mathrm{a}_{2}^{\prime \prime}\right)$, asymmetric stretch $\left(\nu_{3 x} / \nu_{3 y}\right.$, $\left.\mathrm{e}^{\prime}\right)$, and asymmetric bend $\left(\nu_{4 x} / \nu_{4 y}, \mathrm{e}^{\prime}\right)$, respectively. The associated numbers of basis functions/DVR grid points are $15,14,17,17,18$, and 18 , which yields total energies converged to better than $10^{-1} \mathrm{~cm}^{-1}$ when compared to a basis with one basis function less in each mode. The degeneracies are reproduced to better than $10^{-2} \mathrm{~cm}^{-1}$ for all degenerate levels. The diagonalization is carried out in the DVR basis and the resulting eigenvectors are transformed back into the FBR, in which the eigenstate composition can be analyzed conveniently in terms of vibrational quanta in each of the modes.

\section{Results and Discussion}

The eigenstates of three isotopologues of the $\mathrm{NO}_{3} \tilde{X}$ state supported by the lowest adiabatic potential of the new diabatic PES model are collected in Tabs. 3, 4 and 5. These computations were carried out by both the time-dependent MCTDH approach and the time-independent DVR method to gain a maximum of information. The obtained energies are in close agreement and the observed minor differences originate 
from the more approximate kinetic energy used for the DVR calculations. Therefore, we only report the energies from the MCTDH computations. $\mathrm{C}_{2 v}$ symmetry operators are implemented which directly yield the characters of the vibrational wave functions. (see Tab. 1 for $\mathrm{C}_{2 v} / \mathrm{D}_{3 h}$ correlations.) Furthermore, the vibrational wave functions of the DVR calculations are transformed back to the oscillator basis which allows the assignment of each state in terms of the underlying normal modes. The tentative assignments from that analysis are also given in the tables. Note, however, that harmonic normal modes quickly loose their significance in strongly anharmonic systems like $\mathrm{NO}_{3}$ and thus the assignment of vibrational states becomes progressively meaningless. Furthermore, the $e^{\prime}$ normal modes of the $\mathrm{NO}_{3}$ ground state are far from the typical asymmetric stretch and bend coordinates as found for the $\mathrm{NO}_{3}^{-}$anion. This was already observed by Stanton[57] and means that particularly for the higher $\nu_{4}$ excitations the assignment of quantum numbers becomes fairly pointless. Eigenstates which cannot be assigned to a clear excitation in terms of normal modes are labeled by $(*)$ in the tables. The new dipole surface for the $\tilde{X}$ state was used to compute the dipole transition probabilities for IR transitions also presented in the tables. These calculations were performed using the DVR method in which it is trivial to evaluate the integrals needed in the evaluation of

$$
P_{f \leftarrow 0}=\left|\left\langle\Psi_{f}|\vec{\mu}(\boldsymbol{Q})| \Psi_{0}\right\rangle\right|^{2} \cdot \delta\left(E_{0}-E_{f}+h \nu\right) .
$$

The IR intensities are proportional to the corresponding transition probabilities.

We first discuss the results for the most abundant ${ }^{14} \mathrm{~N}^{16} \mathrm{O}_{3}$ isotopologues. The first seven computed eigenstates are in agreement with all experimental and previous theoretical results. The lowest excited state is assigned to $4^{1}$ and is computed at $361 \mathrm{~cm}^{-1}$ in excellent agreement with high-resolution IR results of $365.5 \mathrm{~cm}^{-1}$.[26] This is by far the strongest of all computed IR transitions. There are two levels corresponding to $4^{2}$ with symmetries $a_{1}^{\prime}$ and $e^{\prime}$ of which only the latter is IR allowed. The computed energy of $742 \mathrm{~cm}^{-1}$ for the $e^{\prime}$ state corresponds reasonably well with the experimental value of $771.8 \mathrm{~cm}^{-1}$.[26] The $a_{1}^{\prime}$ level also has been detected at $751.8 \mathrm{~cm}^{-1}$ and is computed at $711 \mathrm{~cm}^{-1}$ in our simulation. [26] The next computed level is found at $748 \mathrm{~cm}^{-1}$ for $2^{1}$ in good agreement with the experimental value at $762.3 \mathrm{~cm}^{-1}$.[26] The state computed at $1021.8 \mathrm{~cm}^{-1}$ can be assigned clearly to the $3^{1}$ fundamental which has been the subject of a long lasting dispute. Our result is in agreement, though found at slightly lower energy, with previous high-level theoretical treatments[34, 57, 59,60] and more recent experimental assignments. [32, 28, 118] We note that the computed IR intensity is the second highest of all states considered here. Nevertheless, it seems that this state was not observed by high-resolution IR spectroscopy. The reason for this is unknown (see discussion below). An experimental value around $1060 \mathrm{~cm}^{-1}$ has been detected by laser induced fluorescence but has been assigned to the $\nu_{1}$ fundamental[19,30]. This is in agreement with the level we compute at $1038.6 \mathrm{~cm}^{-1}$. However, this $1^{1}$ transition is of $a_{1}^{\prime}$ symmetry and thus IR inactive as can be seen from our intensity calculations. The $\nu_{1}$ fundamental is followed by the three levels corresponding to $4^{3}$ between 1082 and $1140 \mathrm{~cm}^{-1}$ of which only the $e^{\prime}$ level is IR active though absorbing much weaker than the previously 
Table 3: Vibrational term values of ${ }^{14} \mathrm{~N}^{16} \mathrm{O}_{3}$, excitation energies in $\mathrm{cm}^{-1}$ with respect to zero-point level $\left(2383.33 \mathrm{~cm}{ }^{-1}\right)$, level symmetries in $C_{2 v}$ and $D_{3 h}$, state assignments, and IR intensities (arbitrary units).

\begin{tabular}{|c|c|c|c|c|c|}
\hline state & $E_{i}-E_{0}$ & $\Gamma_{C_{2 v}}$ & $\Gamma_{D_{3 h}}$ & assignment & IR intensity \\
\hline 1 & 0.0 & $a_{1}$ & $a_{1}^{\prime}$ & 0 & \\
\hline $2 / 3$ & 361.1 & $b_{1} / a_{1}$ & $e^{\prime}$ & $4^{1}$ & $0.778 \times 10^{-02}$ \\
\hline 4 & 711.0 & $a_{1}$ & $a_{1}^{\prime}$ & $4^{2}$ & \\
\hline $5 / 6$ & 742.2 & $b_{1} / a_{1}$ & $e^{\prime}$ & $4^{2}$ & $0.448 \times 10^{-03}$ \\
\hline 7 & 748.5 & $b_{2}$ & $a_{2}^{\prime \prime}$ & $2^{1}$ & $0.860 \times 10^{-03}$ \\
\hline $8 / 9$ & 1021.8 & $a_{1} / b_{1}$ & $e^{\prime}$ & $3^{1}$ & $0.135 \times 10^{-02}$ \\
\hline 10 & 1038.6 & $a_{1}$ & $a_{1}^{\prime}$ & $1^{1}$ & \\
\hline $11 / 12$ & 1082.5 & $b_{1} / a_{1}$ & $e^{\prime}$ & $4^{3}$ & $0.173 \times 10^{-04}$ \\
\hline $13 / 14$ & 1109.6 & $a_{2} / b_{2}$ & $e^{\prime \prime}$ & $2^{1} 4^{1}$ & \\
\hline 15 & 1134.6 & $b_{1}$ & $a_{2}^{\prime}$ & $4^{3}$ & \\
\hline 16 & 1139.7 & $a_{1}$ & $a_{1}^{\prime}$ & $4^{3}$ & \\
\hline 17 & 1302.4 & $b_{1}$ & $a_{2}^{\prime}$ & $3^{1} 4^{1}$ & \\
\hline $18 / 19$ & 1388.1 & $a_{1} / b_{1}$ & $e^{j}$ & $1^{1} 4^{1}$ & $0.280 \times 10^{-03}$ \\
\hline 20 & 1425.0 & $a_{1}$ & $a_{1}^{\prime}$ & $(*)$ & \\
\hline $21 / 22$ & 1438.6 & $a_{1} / b_{1}$ & $e^{\prime}$ & $3^{1} 4^{1}$ & $0.939 \times 10^{-03}$ \\
\hline 23 & 1446.9 & $a_{1}$ & $a_{1}^{\prime}$ & $4^{4} / 4^{5}$ & \\
\hline 24 & 1459.2 & $b_{2}$ & $a_{2}^{\prime \prime}$ & $2^{1} 4^{2}$ & $0.209 \times 10^{-07}$ \\
\hline $25 / 26$ & 1469.1 & $b_{1} / a_{1}$ & $e^{\prime}$ & $4^{4}$ & $0.381 \times 10^{-04}$ \\
\hline $27 / 28$ & 1490.2 & $a_{2} / b_{2}$ & $e^{\prime \prime}$ & $2^{1} 4^{2}$ & \\
\hline 29 & 1496.2 & $a_{1}$ & $a_{1}^{\prime}$ & $2^{2}$ & \\
\hline $30 / 31$ & 1542.0 & $a_{1} / b_{1}$ & $e^{\prime}$ & $4^{4}$ & $0.249 \times 10^{-05}$ \\
\hline $32 / 33$ & 1678.2 & $a_{1} / b_{1}$ & $e^{\prime}$ & $3^{1} 4^{2}$ & $0.369 \times 10^{-03}$ \\
\hline 34 & 1736.4 & $a_{1}$ & $a_{1}^{\prime}$ & $1^{1} 4^{2}$ & \\
\hline $35 / 36$ & 1763.1 & $b_{2} / a_{2}$ & $e^{\prime \prime}$ & $2^{1} 3^{1}$ & \\
\hline $37 / 38$ & 1772.6 & $b_{1} / a_{1}$ & $e^{\prime}$ & $1^{1} 4^{2}$ & $0.760 \times 10^{-04}$ \\
\hline 39 & 1783.5 & $b_{2}$ & $a_{2}^{\prime \prime}$ & $1^{1} 2^{1}$ & $0.240 \times 10^{-05}$ \\
\hline 40 & 1803.9 & $b_{1}$ & $a_{2}^{\prime}$ & $3^{1} 4^{2} / 3^{1} 4^{3}$ & \\
\hline $41 / 42$ & 1810.7 & $a_{1} / b_{1}$ & $e^{i}$ & $(*)$ & $0.187 \times 10^{-04}$ \\
\hline $43 / 44$ & 1830.1 & $a_{2} / b_{2}$ & $e^{\prime \prime}$ & $2^{1} 4^{3}$ & \\
\hline $45 / 46$ & 1831.2 & $a_{1} / b_{1}$ & $e^{\prime}$ & $(*)$ & $0.916 \times 10^{-05}$ \\
\hline $47 / 48$ & 1857.3 & $b_{1} / a_{1}$ & $e^{\prime}$ & $2^{2} 4^{1}$ & $0.458 \times 10^{-06}$ \\
\hline 49 & 1860.4 & $b_{1}$ & $a_{2}^{\prime}$ & $(*)$ & \\
\hline 50 & 1861.3 & $a_{1}$ & $a_{1}^{\prime}$ & $(*)$ & \\
\hline 51 & 1881.7 & $a_{1}$ & $a_{1}^{\prime}$ & $(*)$ & \\
\hline 52 & 1882.0 & $a_{2}$ & $a_{1}^{\prime \prime}$ & $2^{1} 4^{3}$ & \\
\hline 53 & 1887.0 & $b_{2}$ & $a_{2}^{\prime \prime}$ & $2^{1} 4^{3}$ & $0.212 \times 10^{-07}$ \\
\hline 54 & 1914.2 & $a_{1}$ & $a_{1}^{\prime}$ & $(*)$ & \\
\hline
\end{tabular}

discussed transitions. The next state with considerable oscillator strength is assigned to $1^{1} 4^{1}$ at $1388 \mathrm{~cm}^{-1}$ which is in reasonable agreement with an LIF feature assigned to this state at $1420 \mathrm{~cm}^{-1}$.[30] The second state strongly debated corresponds to $3^{1} 4^{1}\left(e^{\prime}\right)$ and is calculated at $1439 \mathrm{~cm}^{-1}$ compared to an experimental 
Table 4: Vibrational term values of ${ }^{15} \mathrm{NO}_{3}$, excitation energies in $\mathrm{cm}^{-1}$ with respect to zero-point (2352.13 $\mathrm{cm}^{-1}$ ), level symmetries in $C_{2 v}$ and $D_{3 h}$, state assignments including dominant state contributions, and IR intensities (arbitrary units).

\begin{tabular}{|c|c|c|c|c|c|}
\hline state & $E_{0}$ or $E_{i}-E_{0}$ & $\Gamma_{C_{2 v}}$ & $\Gamma_{D_{3 h}}$ & assignment & IR intensity \\
\hline 1 & 0.0 & $a_{1}$ & $a_{1}^{\prime}$ & 0 & \\
\hline $2 / 3$ & 356.3 & $b_{1} / a_{1}$ & $e^{\prime}$ & $4^{1}$ & $0.772 \times 10^{-02}$ \\
\hline 4 & 702.5 & $a_{1}$ & $a_{1}^{\prime}$ & $4^{2}$ & \\
\hline 5 & 729.2 & $b_{2}$ & $a_{2}^{\prime \prime}$ & $2^{1}$ & $0.837 \times 10^{-03}$ \\
\hline $6 / 7$ & 732.2 & $b_{1} / a_{1}$ & $e^{\prime}$ & $(*)$ & $0.461 \times 10^{-03}$ \\
\hline $8 / 9$ & 1008.4 & $a_{1} / b_{1}$ & $e^{\prime}$ & $3^{1}$ & $0.120 \times 10^{-02}$ \\
\hline 10 & 1038.1 & $a_{1}$ & $a_{1}^{\prime}$ & $1^{1}$ & \\
\hline $11 / 12$ & 1069.6 & $b_{1} / a_{1}$ & $e^{\prime}$ & $(*)$ & $0.147 \times 10^{-04}$ \\
\hline $13 / 14$ & 1085.5 & $a_{2} / b_{2}$ & $e^{\prime \prime}$ & $2^{1} 4^{1}$ & \\
\hline 15 & 1118.8 & $b_{1}$ & $a_{2}^{\prime}$ & $4^{3}(?)$ & \\
\hline 16 & 1124.8 & $a_{1}$ & $a_{1}^{\prime}$ & $4^{3}(?)$ & \\
\hline 17 & 1287.0 & $b_{1}$ & $a_{2}^{\prime}$ & $3^{1} 4^{1}$ & \\
\hline $18 / 19$ & 1380.4 & $a_{1} / b_{1}$ & $e^{\prime}$ & $1^{1} 4^{1}$ & $0.394 \times 10^{-03}$ \\
\hline 20 & 1404.1 & $a_{1}$ & $a_{1}^{\prime}$ & $(*)$ & \\
\hline $21 / 22$ & 1420.4 & $a_{1} / b_{1}$ & $e^{\prime}$ & $(*)$ & $0.811 \times 10^{-03}$ \\
\hline 23 & 1431.1 & $a_{1}$ & $a_{1}^{\prime}$ & $(*)$ & \\
\hline 24 & 1431.4 & $b_{2}$ & $a_{2}^{\prime \prime}$ & $(*)$ & $0.203 \times 10^{-07}$ \\
\hline $25 / 26$ & 1452.6 & $b_{1} / a_{1}$ & $e^{\prime}$ & $(*)$ & $0.717 \times 10^{-04}$ \\
\hline 27 & 1457.6 & $a_{1}$ & $a_{1}^{\prime}$ & $2^{2}$ & \\
\hline $28 / 29$ & 1460.9 & $a_{2} / b_{2}$ & $e^{\prime \prime}$ & $(*)$ & \\
\hline $30 / 31$ & 1521.1 & $a_{1} / b_{1}$ & $e^{\prime}$ & $(*)$ & $0.433 \times 10^{-05}$ \\
\hline $32 / 33$ & 1658.6 & $a_{1} / b_{1}$ & $e^{\prime}$ & $(*)$ & $0.376 \times 10^{-03}$ \\
\hline 34 & 1726.8 & $a_{1}$ & $a_{1}^{\prime}$ & $(*)$ & \\
\hline $35 / 36$ & 1730.8 & $b_{2} / a_{2}$ & $e^{\prime \prime}$ & $(*)$ & \\
\hline $37 / 38$ & 1758.2 & $a_{1} / b_{1}$ & $e^{\prime}$ & $(*)$ & $0.550 \times 10^{-04}$ \\
\hline 39 & 1763.7 & $b_{2}$ & $a_{2}^{\prime \prime}$ & $1^{1} 2^{1}$ & $0.232 \times 10^{-05}$ \\
\hline 40 & 1780.1 & $b_{1}$ & $a_{2}^{\prime}$ & $(*)$ & \\
\hline $41 / 42$ & 1784.9 & $a_{1} / b_{1}$ & $e^{\prime}$ & $(*)$ & $0.273 \times 10^{-04}$ \\
\hline $43 / 44$ & 1798.1 & $a_{2} / b_{2}$ & $e^{\prime \prime}$ & (*) & \\
\hline $45 / 45$ & 1811.0 & $a_{1} / b_{1}$ & $e^{\prime}$ & $2^{2} 4^{1}(*)$ & $0.446 \times 10^{-05}$ \\
\hline $47 / 48$ & 1815.1 & $a_{1} / b_{1}$ & $e^{\prime}$ & $2^{2} 4^{1}(*)$ & $0.691 \times 10^{-05}$ \\
\hline 49 & 1836.0 & $a_{1}$ & $a_{1}^{\prime}$ & $(*)$ & \\
\hline 50 & 1838.6 & $b_{1}$ & $a_{2}^{\prime}$ & $(*)$ & \\
\hline 51 & 1846.9 & $a_{2}$ & $a_{1}^{\prime \prime}$ & $(*)$ & \\
\hline 52 & 1852.9 & $b_{2}$ & $a_{2}^{\prime \prime}$ & $(*)$ & $0.382 \times 10^{-07}$ \\
\hline
\end{tabular}

band at $1492 \mathrm{~cm}^{-1}$. We compute the third strongest IR intensity for this transition and cannot find any other strong transition in that energy region. Thus, our result clearly supports the assignment of the $1492 \mathrm{~cm}^{-1}$ feature to the $3^{1} 4^{1}\left(e^{\prime}\right)$ transition. The $3^{1} 4^{1}$ configuration gives rise to three sub-levels of $e^{\prime}$, $a_{1}^{\prime}$, and $a_{2}^{\prime}$ symmetry. The $a_{2}^{\prime}$ level corresponding to $3^{1} 4^{1}$ is found at much lower energy at $1302 \mathrm{~cm}^{-1}$ which is rather unexpected and might indicate some remaining problems with the PES model. The $a_{1}^{\prime}$ level 
Table 5: Vibrational term values of ${ }^{14} \mathrm{~N}^{18} \mathrm{O}^{16} \mathrm{O}_{2}$, excitation energies in $\mathrm{cm}^{-1}$ with respect to zero-point level (2355.5 $\mathrm{cm}{ }^{-1}$ ), level symmetries in $C_{2 v}$, state assignments including dominant state contributions, and IR intensities (arbitrary units).

\begin{tabular}{|c|c|c|c|c|}
\hline state & $E_{i}-E_{0}$ & $\Gamma_{C_{2 v}}$ & assignment & IR intensity \\
\hline 1 & 0.0 & $a_{1}$ & 0 & \\
\hline 2,3 & $354.0,358.9$ & $b_{1}, a_{1}$ & $4_{y}^{1}, 4_{x}^{1}$ & $0.383 \times 10^{-02}, 0.383 \times 10^{-02}$ \\
\hline 4 & 700.8 & $a_{1}$ & $4_{u}^{2}$ & $0.582 \times 10^{-05}$ \\
\hline 5,6 & $732.4,733.8$ & $b_{1}, a_{1}$ & $4_{x}^{1} 4_{y}^{1}, 4_{x}^{2}$ & $0.207 \times 10^{-03}, 0.207 \times 10^{-03}$ \\
\hline 7 & 745.4 & $b_{2}$ & $2^{1}$ & $0.858 \times 10^{-03}$ \\
\hline 8,9 & $993.9,1017.9$ & $a_{1}, b_{1}$ & $3_{x}^{1}, 3_{u}^{1}$ & $0.474 \times 10^{-03}, 0.738 \times 10^{-03}$ \\
\hline 10 & 1030.5 & $a_{1}$ & $1^{1}$ & $0.216 \times 10^{-03}$ \\
\hline 11,12 & $1062.5,1072.7$ & $b_{1}, a_{1}$ & $4_{u}^{3}, 4_{x}^{3}$ & $0.825 \times 10^{-05}, 0.905 \times 10^{-05}$ \\
\hline 13,14 & $1099.5,1104.2$ & $a_{2}, b_{2}$ & $2^{1} 4_{y}^{1}, 2^{1} 4_{x}^{1}$ & $-\quad, 0.104 \times 10^{-08}$ \\
\hline 15 & 1120.5 & $b_{1}$ & $4_{x}^{2} 4_{y}^{1}$ & $0.427 \times 10^{-07}$ \\
\hline 16 & 1125.2 & $a_{1}$ & $4_{x}^{1} 4_{y}^{2}$ & $0.539 \times 10^{-06}$ \\
\hline 17 & 1285.4 & $b_{1}$ & $3_{x}^{1} 4_{u}^{1} / 3_{u}^{1} 4_{x}^{1}$ & $0.560 \times 10^{-05}$ \\
\hline 18,19 & $1358.6,1372.0$ & $b_{1}, a_{1}$ & $1^{1} 4_{y}^{1}, 1^{1} 4_{x}^{1}$ & $0.144 \times 10^{-03}, 0.350 \times 10^{-04}$ \\
\hline 20 & 1407.8 & $a_{1}$ & $3_{x}^{1} 4_{x}^{1}$ & $0.265 \times 10^{-03}$ \\
\hline 21 & 1418.0 & $a_{1}$ & $3_{u}^{1} 4_{u}^{1}$ & $0.245 \times 10^{-03}$ \\
\hline 22 & 1425.9 & $a_{1}$ & $(*)$ & $0.539 \times 10^{-04}$ \\
\hline 23 & 1430.1 & $b_{1}$ & $(*)$ & $0.411 \times 10^{-03}$ \\
\hline 24 & 1446.0 & $b_{2}$ & $2^{1} 4_{y}^{2} / 2^{1} 4_{x}^{2}$ & $0.195 \times 10^{-07}$ \\
\hline 25,26 & $1449.2,1451.5$ & $b_{1}, a_{1}$ & $(*),(*)^{*}$ & $0.321 \times 10^{-04}, 0.371 \times 10^{-05}$ \\
\hline 27,28 & $1477.3,1478.7$ & $a_{2}, b_{2}$ & $2^{1} 4_{x}^{1} 4_{y}^{1}, 2^{1} 4_{x}^{2}$ & $-\quad, 0.361 \times 10^{-09}$ \\
\hline 29 & 1490.1 & $a_{1}$ & $2^{2}$ & $0.214 \times 10^{-06}$ \\
\hline 30,31 & $1522.1,1522.9$ & $b_{1}, a_{1}$ & $4_{x}^{3} 4_{y}^{1} / 4_{x}^{1} 4_{y}^{3}, 4_{x}^{2} 4_{y}^{2}$ & $0.158 \times 10^{-06}, 0.641 \times 10^{-06}$ \\
\hline 32,33 & $1646.8,1666.4$ & $a_{1}, b_{1}$ & $3_{x}^{1} 4_{y}^{2}, 3_{y}^{1} 4_{x}^{2}$ & $0.166 \times 10^{-03}, 0.180 \times 10^{-03}$ \\
\hline 34 & 1709.5 & $a_{1}$ & $1^{1} 4_{y}^{2} / 1^{1} 4_{x}^{2}$ & $0.725 \times 10^{-05}$ \\
\hline 35,37 & $1733.7,1754.9$ & $b_{2}, a_{2}$ & $2^{1} 3_{x}^{1} / 1^{1} 2^{1}, 2^{1} 3_{y}^{1}$ & $0.590 \times 10^{-06}, \quad-$ \\
\hline 36,38 & $1736.2,1755.9$ & $b_{1}, a_{1}$ & $1^{1} 4_{x}^{1} 4_{y}^{1}, 1^{1} 4_{x}^{2} / 1^{1} 4_{y}^{2}$ & $0.231 \times 10^{-04}, 0.556 \times 10^{-04}$ \\
\hline 39 & 1771.2 & $b_{2}$ & $1^{1} 2^{1}$ & $0.176 \times 10^{-05}$ \\
\hline 40 & 1783.5 & $b_{1}$ & $3_{x}^{1} 4_{y}^{3}$ & $0.187 \times 10^{-07}$ \\
\hline 41,42 & $1786.8,1790.3$ & $b_{1}, a_{1}$ & $(*),(*)$ & $0.355 \times 10^{-05}, 0.335 \times 10^{-05}$ \\
\hline 43,46 & $1807.3,1817.2$ & $a_{2}, b_{2}$ & $2^{1} 4_{u}^{3}, 2^{1} 4_{x}^{3}(*)$ & $-\quad, 0.213 \times 10^{-08}$ \\
\hline 44,45 & $1807.9,1808.0$ & $a_{1}, b_{1}$ & $(*),(*)$ & $0.183 \times 10^{-05}, 0.156 \times 10^{-04}$ \\
\hline 47 & 1836.0 & $b_{1}$ & $(*)$ & $0.113 \times 10^{-06}$ \\
\hline 48 & 1839.7 & $a_{1}$ & $(*)$ & $0.663 \times 10^{-07}$ \\
\hline 49,50 & $1844.3,1848.6$ & $b_{1}, a_{1}$ & $2^{2} 4_{y}^{1}, 2^{2} 4_{x}^{1}$ & $0.296 \times 10^{-06}, 0.349 \times 10^{-06}$ \\
\hline 51 & 1856.1 & $a_{1}$ & $(*)$ & $0.366 \times 10^{-05}$ \\
\hline 52 & 1864.9 & $a_{2}$ & $2^{1} 4_{x}^{2} 4_{y}^{1}$ & - \\
\hline 53 & 1869.4 & $b_{2}$ & $2^{1} 4_{x}^{1} 4_{y}^{2}(*)$ & $0.151 \times 10^{-07}$ \\
\hline
\end{tabular}

cannot be clearly assigned to any of the states analysed here. Possible candidates in reasonable energetic proximity would be the levels computed at 1425.0 or $1446.9 \mathrm{~cm}^{-1}$. The decomposition of the computed level at $1425 \mathrm{~cm}^{-1}$ of $a_{1}^{\prime}$ symmetry into the harmonic oscillator basis shows a strong mixing of excitations in terms of normal modes, which does not allow for a clear assignment. It does contain some contributions of $3^{1} 4^{1}$ 
Table 6: Summary of the comparison between experimental and computed transition energies for $\mathrm{NO}_{3}$ (in $\mathrm{cm}^{-1}$ ). Experimental data as summarized in ref. [118]. See text for discussion.

\begin{tabular}{cccc|c|cc} 
assignment & $E_{\text {exp }}$ & $E_{\text {theo }}$ & $\mathrm{IR}$ & $E_{\text {exp }}-E_{\text {theo }}$ & ${ }^{15} \Delta_{\text {exp }}$ & ${ }^{15} \Delta_{\text {theo }}$ \\
\hline $4^{1} e^{\prime}$ & 365.5 & 361.1 & $0.778 \times 10^{-02}$ & 4.4 & -5.3 & -4.8 \\
$4^{2} a_{1}^{\prime}$ & 752.4 & 711.0 & & 41.4 & -9.8 & -8.5 \\
$4^{2} e^{\prime}$ & 771.8 & 742.2 & $0.448 \times 10^{-03}$ & 29.6 & -10.6 & -10.0 \\
$2^{1} a_{2}^{\prime \prime}$ & 762.3 & 748.5 & $0.860 \times 10^{-03}$ & 13.8 & -19.6 & -19.3 \\
$1^{1} a_{1}^{\prime}$ & 1051.2 & 1038.6 & & 12.6 & 1.5 & -0.5 \\
$3^{1} e^{\prime}$ & 1055.3 & 1021.8 & $0.135 \times 10^{-02}$ & 33.5 & -16.7 & -13.4 \\
$2^{1} 4^{1} e^{\prime \prime}$ & 1125.1 & 1109.6 & & 15.5 & -25.1 & -24.1 \\
$4^{3} e^{\prime}$ & 1173.6 & 1082.5 & $0.173 \times 10^{-04}$ & 91.1 & -14.4 & -12.9 \\
$4^{3} a_{1}^{\prime}$ & 1214 & 1139.7 & & 74.3 & -15.0 & -14.9 \\
$1^{1} 4^{1} e^{\prime}$ & 1413.6 & 1388.1 & $0.280 \times 10^{-03}$ & 25.5 & -6.5 & -7.7 \\
$3^{1} 4^{1} a_{2}^{\prime}$ & 1491 & $1302.4(?)$ & & $188.6(?)$ & -26.0 & -15.4 \\
$3^{1} 4^{1} e^{\prime}$ & 1492.4 & 1438.6 & $0.939 \times 10^{-03}$ & 53.8 & -19.6 & -18.2 \\
$3^{1} 4^{1} a_{1}^{\prime}$ & 1499.8 & - & & - & -24.6 & \\
$2^{1} 4^{2} a_{2}^{\prime \prime}$ & 1509.7 & 1459.2 & $0.209 \times 10^{-07}$ & 50.5 & -29.6 & -27.8 \\
$2^{2} a_{1}^{\prime}$ & 1522 & 1496.2 & & 25.8 & -39.0 & -38.6 \\
$2^{1} 4^{2} e^{\prime \prime}$ & 1537.5 & 1490.2 & & 47.3 & -5.5 & -29.3 \\
$1^{1} 4^{2} a_{1}^{\prime}$ & 1773.8 & 1736.4 & & 37.4 & -10.0 & -9.6 \\
$1^{1} 4^{2} e^{\prime}$ & 1793 & 1772.6 & $0.760 \times 10^{-04}$ & 20.4 & -10.0 & -14.4 \\
$1^{1} 2^{1} a_{2}^{\prime \prime}$ & 1810 & 1783.5 & $0.240 \times 10^{-05}$ & 26.5 & -20.0 & -19.8 \\
$2^{1} 3^{1} e^{\prime \prime}$ & 1815 & 1763.1 & & 51.9 & -40.0 & -32.3 \\
$2^{2} 4^{1} e^{\prime}$ & 1885 & 1857.3 & $0.458 \times 10^{-06}$ & 27.7 & -45.0 & -42.2 \\
$2^{1} 4^{3} e^{\prime \prime}$ & 1929.4 & 1830.1 & & 99.3 & -33.6 & -32.0 \\
$2^{1} 4^{3} a_{1}^{\prime \prime}$ & 1937.7 & 1882.0 & & 55.7 & -30.4 & -35.1 \\
$2^{1} 4^{3} a_{2}^{\prime \prime}$ & 1970 & 1887.7 & $0.212 \times 10^{-07}$ & 82.3 & -34.0 & -34.1
\end{tabular}


Table 7: Contributions of diabatic electronic states to low energy vibronic eigenstates.

\begin{tabular}{rrcccc} 
Energy & $\tilde{X}^{2} A_{2}^{\prime}$ & $\tilde{A}^{2} E_{x}^{\prime \prime}$ & $\tilde{A}^{2} E_{y}^{\prime \prime}$ & $\tilde{B}^{2} E_{x}^{\prime}$ & $\tilde{B}^{2} E_{y}^{\prime}$ \\
\hline 0.0 & 0.976 & $0.3 \times 10^{-9}$ & $0.3 \times 10^{-9}$ & $0.12 \times 10^{-1}$ & $0.12 \times 10^{-1}$ \\
361.1 & 0.966 & $0.3 \times 10^{-9}$ & $0.3 \times 10^{-9}$ & $0.17 \times 10^{-1}$ & $0.17 \times 10^{-1}$ \\
361.1 & 0.966 & $0.3 \times 10^{-9}$ & $0.3 \times 10^{-9}$ & $0.17 \times 10^{-1}$ & $0.17 \times 10^{-1}$ \\
711.0 & 0.958 & $0.3 \times 10^{-9}$ & $0.3 \times 10^{-9}$ & $0.21 \times 10^{-1}$ & $0.21 \times 10^{-1}$ \\
742.2 & 0.958 & $0.4 \times 10^{-9}$ & $0.4 \times 10^{-9}$ & $0.21 \times 10^{-1}$ & $0.21 \times 10^{-1}$ \\
742.2 & 0.958 & $0.4 \times 10^{-9}$ & $0.4 \times 10^{-9}$ & $0.21 \times 10^{-1}$ & $0.21 \times 10^{-1}$ \\
748.5 & 0.977 & $0.8 \times 10^{-9}$ & $0.8 \times 10^{-9}$ & $0.12 \times 10^{-1}$ & $0.12 \times 10^{-1}$ \\
1021.8 & 0.957 & $0.1 \times 10^{-8}$ & $0.1 \times 10^{-8}$ & $0.21 \times 10^{-1}$ & $0.21 \times 10^{-1}$ \\
1021.8 & 0.957 & $0.1 \times 10^{-8}$ & $0.1 \times 10^{-8}$ & $0.21 \times 10^{-1}$ & $0.21 \times 10^{-1}$ \\
1038.6 & 0.972 & $0.4 \times 10^{-9}$ & $0.4 \times 10^{-9}$ & $0.14 \times 10^{-1}$ & $0.14 \times 10^{-1}$ \\
1082.5 & 0.952 & $0.3 \times 10^{-9}$ & $0.3 \times 10^{-9}$ & $0.24 \times 10^{-1}$ & $0.24 \times 10^{-1}$ \\
1082.5 & 0.952 & $0.3 \times 10^{-9}$ & $0.3 \times 10^{-9}$ & $0.24 \times 10^{-1}$ & $0.24 \times 10^{-1}$ \\
1109.6 & 0.966 & $0.1 \times 10^{-8}$ & $0.9 \times 10^{-9}$ & $0.21 \times 10^{-1}$ & $0.13 \times 10^{-1}$ \\
1109.6 & 0.966 & $0.9 \times 10^{-9}$ & $0.1 \times 10^{-8}$ & $0.13 \times 10^{-1}$ & $0.21 \times 10^{-1}$ \\
1134.6 & 0.953 & $0.4 \times 10^{-9}$ & $0.4 \times 10^{-9}$ & $0.23 \times 10^{-1}$ & $0.23 \times 10^{-1}$ \\
1139.7 & 0.951 & $0.4 \times 10^{-9}$ & $0.4 \times 10^{-9}$ & $0.25 \times 10^{-1}$ & $0.25 \times 10^{-1}$ \\
1302.4 & 0.945 & $0.1 \times 10^{-8}$ & $0.1 \times 10^{-8}$ & $0.27 \times 10^{-1}$ & $0.27 \times 10^{-1}$ \\
1388.1 & 0.959 & $0.6 \times 10^{-9}$ & $0.6 \times 10^{-9}$ & $0.20 \times 10^{-1}$ & $0.21 \times 10^{-1}$ \\
1388.1 & 0.959 & $0.6 \times 10^{-9}$ & $0.6 \times 10^{-9}$ & $0.21 \times 10^{-1}$ & $0.20 \times 10^{-1}$ \\
1425.0 & 0.948 & $0.2 \times 10^{-8}$ & $0.2 \times 10^{-8}$ & $0.26 \times 10^{-1}$ & $0.26 \times 10^{-1}$ \\
\hline
\end{tabular}

configurations, though. Comparison with the experimental assignment[27] would make the next level about $8 \mathrm{~cm}^{-1}$ above at $1446.9 \mathrm{~cm}^{-1}$ a likely candidate but this state is mostly composed of $4^{4}$ and $4^{5}$ configurations in disagreement with the interpretation of the experimental data. Tab. 6 gathers the comparisons discussed above between experimental frequencies as summarized in ref. [118] and our theoretical results.

The level energies for the higher states are in less good agreement with experimental assignments and the analysis in terms of normal modes is getting progressively more uncertain. It is also worth noting that the different high-level ab initio quantum dynamics calculations of the vibrational level energies (and assignments) all deviate considerably, even among studies by the same authors.[34, 57, 59, 60] One possible reason could be the treatment of the vibronic coupling to the $\tilde{B}$ state, which is also invoked for the explanation of various perturbations observed in the high-resolution spectra. We therefore tested the role of the $\tilde{B}$ state by a number of calculations. First of all, we computed the contributions of the five diabatic electronic states to the lowest 20 eigenstates (Tab. 7). We computed the eigenstates using both the adiabatic as well as the diabatic electronic basis and found basically no difference in the level energies. The diabatic contributions are obtained directly from the normalized DVR state eigenvectors of the diabatic calculation as norms of the five sub-vectors each belonging to one of the five diabatic electronic states. As is not surprising, all states are by far dominated by the $\tilde{X}$ state contribution and the $\tilde{A}$ state has a negligible effect. The $\tilde{B}$ state contributes between $2-5 \%$ to the total state composition with a very slight tendency to mix in increasingly 
with higher vibrational level energy as would be expected. These results can be used also to test arguments in the debate about the assignment of the experimental $1492 \mathrm{~cm}^{-1}$ band. In the debate between the groups of Hirota[119] and Kawaguchi[28] the vibronic coupling between the $\tilde{X}$ and $\tilde{B}$ state plays a key role. One argument concerns the observed effective spin-orbit (SO) coupling in the non-degenerate ${ }^{2} A_{2}^{\prime}$ ground state that can only originate from a contribution by a degenerate excited electronic state. We computed the SO splittings of the excited states at MRCI level of theory and found values of $-1 \mathrm{~cm}^{-1}$ for the $\tilde{A}^{2} E^{\prime \prime}$ state and $-107 \mathrm{~cm}^{-1}$ for the $\tilde{B}^{2} E^{\prime}$ state, respectively. The latter is in excellent agreement with the experimental estimate of $-105 \mathrm{~cm}^{-1}$ by Tada et al.[120] The value of $-105 \mathrm{~cm}^{-1}$ corresponds to an effective SO coupling constant of $-21 \mathrm{~cm}^{-1}$ and a contribution of $5 \% \tilde{B}$ state to the $\tilde{X}$ state vibronic levels would correspond to an effective SO coupling constant of $-1.05 \mathrm{~cm}^{-1}$ for $\tilde{X}$ state levels in stark contrast to the experimental value of $-0.167 \mathrm{~cm}^{-1}$.

The coupling between the $\tilde{X}$ and the $\tilde{B}$ state has also been invoked to explain anomalies in line intensities in both IR spectra and the photodetachment spectrum of the $\mathrm{NO}_{3}^{-}$anion.[27, 59] Stanton computed the IR absorption intensity for the $3^{1}$ fundamental to be very low despite it being an allowed $e^{\prime}$ level and the asymmetric stretching mode inducing a significant change in dipole moment. [57] This result cannot be reproduced by our current model for the PES and the dipole surface. The pronounced non-linearity and multi-mode dependence of the dipole surface that we observe may indicate the influence of the vibronic $\tilde{X}-\tilde{B}$ coupling. However, this dipole surface results in a considerable IR intensity for the $\nu_{3}$ fundamental in contrast to experimental observations. Kawaguchi et al. suggested that the sizable coupling to the $\tilde{B}$ state together with the large $\tilde{X}$ - $\tilde{B}$ transition moment leads to intensity cancellation for the $3^{1}$ transition.[27] This seems a reasonable explanation. The numerical test of this hypothesis is beyond the scope of the present study but will be investigated in the near future.

The vibrational eigenstates for the $\tilde{X}$ state have been calculated as well for two isotopologues, namely ${ }^{15} \mathrm{~N}^{16} \mathrm{O}_{3}$ and ${ }^{14} \mathrm{~N}^{18} \mathrm{O}^{16} \mathrm{O}_{2}$, and the corresponding results are summarized in Tabs. 4 and 5, respectively. For ${ }^{15} \mathrm{~N}^{16} \mathrm{O}_{3}$, the system remains $D_{3 h}$ symmetric and thus the vibrational states have the same symmetry labels as for the natural isotopologues. By contrast, ${ }^{14} \mathrm{~N}^{18} \mathrm{O}^{16} \mathrm{O}_{2}$ only has $C_{2 v}$ symmetry and thus all levels corresponding to $e^{\prime}$ and $e^{\prime \prime}$ symmetry for ${ }^{14} \mathrm{~N}^{16} \mathrm{O}_{3}$ and ${ }^{15} \mathrm{~N}^{16} \mathrm{O}_{3}$ will split into $a_{1} / b_{1}$ and $a_{2} / b_{2}$ levels, respectively. Experimental values are available for the isotopic shifts of some of the vibrational levels. Beckers et al. studied isotopic shifts of ${ }^{15} \mathrm{~N}^{16} \mathrm{O}_{3}$ in a Ne matrix at $4.3 \mathrm{~K}$ and for the first time observed the shifts for the $4^{1}$ state of $-5.3 \mathrm{~cm}^{-1}$, the $3^{1}$ state of $-12.8 \mathrm{~cm}^{-1}$, and the $1^{1} 4^{1}$ state of $-6.6 \mathrm{~cm}^{-1}$.[33] The corresponding computed values are $-4.8 \mathrm{~cm}^{-1},-13.4 \mathrm{~cm}^{-1}$, and $-7.7 \mathrm{~cm}^{-1}$, respectively, in very good agreement with experiment (c. f. Tab. 6). Fujimori et al. studied the same isotopologue and thoroughly investigated three states.[26] The $2^{1}$ level shows an isotopic shift of $-19.6 \mathrm{~cm}^{-1}$ in excellent agreement with our computed value of $-19.3 \mathrm{~cm}^{-1}$. For the two states corresponding to $4^{2}$ excitation the experimental values are $-9.2 \mathrm{~cm}^{-1}$ for the $a_{1}^{\prime}$ level and $-10.5 \mathrm{~cm}^{-1}$ for the $e^{\prime}$ state. Our corresponding computed values are 
$-8.5 \mathrm{~cm}^{-1}$ and $-10.0 \mathrm{~cm}^{-1}$, both in excellent agreement with experiment. The isotopic shifts of three other levels have been determined experimentally by Kawaguchi et al., most importantly the $1492 \mathrm{~cm}^{-1}$ feature under debate.[27] They observed a shift of $-19.6 \mathrm{~cm}^{-1}$ for the $e^{\prime}$ state corresponding to $3^{1} 4^{1}$ excitation in nearly perfect agreement with our computation of $-19.3 \mathrm{~cm}^{-1}$. By contrast, they assign a feature at $1499 \mathrm{~cm}^{-1}$ to the $a_{1}^{\prime}$ state for the same excitation with an isotopic shift of $-24.6 \mathrm{~cm}^{-1}$ which is not found in our simulations. We find a state $8 \mathrm{~cm}^{-1}$ above the $3^{1} 4^{1} e^{\prime}$ state at $1447 \mathrm{~cm}^{-1}$ but its isotopic shift is only $-15.6 \mathrm{~cm}^{-1}$. We also compute a $2^{2} a_{1}^{\prime}$ state at $1497 \mathrm{~cm}^{-1}$ with a shift of $-38.6 \mathrm{~cm}^{-1}$ which can be ruled out as well. Another candidate with $a_{1}^{\prime}$ symmetry cannot be clearly assigned from our calculations and is computed at $1425 \mathrm{~cm}^{-1}$ with a shift of $-20.9 \mathrm{~cm}^{-1}$. This represents the closest match in the isotopic shift though the computed level energies is in less good agreement with experiment.

Finally, Kawaguchi et al. assigned a feature at $1557.3 \mathrm{~cm}^{-1}$ to the $2^{1} 4^{2} e^{\prime \prime}$ state and determined an isotopic shift of $-5.5 \mathrm{~cm}^{-1}$. The present PES model yields a calculated transition at $1490.2 \mathrm{~cm}^{-1}$ with a corresponding shift of $-29.6 \mathrm{~cm}^{-1}$ for the same state. The computed isotopic shifts of all undisputed states are all within $2 \mathrm{~cm}^{-1}$ of the experimental values. Therefore, we can use the isotopic shifts as indicator for inconsistencies in the assignments between theory and experiment. Kawaguchi et al. recently presented a summary of level energies, assignments, and isotopic shift, which we use in Table 6 to compare to the data obtained in the present work. We notice that particularly two levels show stark discrepancies between our results and Kawaguchi's assignments, namely the $3^{1} 4^{1}\left(a_{2}^{\prime}\right)$ level and the $2^{1} 4^{2}\left(e^{\prime \prime}\right)$ state. In the first case our result for the shift is lower than the sum of the single mode shifts while the opposite is true for the experimental assignment. For the above mentioned $e^{\prime \prime}$ state the experimental result seems rather inconsistent with the single mode shifts while the theoretical result seems to match perfectly. Therefore, a re-investigation of these particular bands might be worthwhile. We also note that of all the fundamentals the $\nu_{3}$ one shows the largest deviation between experimental and theoretical isotope shift. The reason for this is unknown but the result indicates a different nuclear motion predicted by theory compared to experiment. Together with the observation that the $\nu_{4}$ progression seems to have the wrong anharmonicity behaviour compared to experiment, rendering level energies involving higher $\nu_{4}$ excitations progressively less accurate, this might indicate some remaining deficiency of the PES model. This will be addressed in forthcoming work.

The results of the simulation of the ${ }^{14} \mathrm{~N}^{18} \mathrm{O}^{16} \mathrm{O}_{2}$ isotopologue are also presented in Tab. 5. Unfortunately, experimental measurements are limited to only two Ne matrix studies $[32,33]$ in which the $4^{1}$ fundamental was observed as two transitions at 361.1 and $357.9 \mathrm{~cm}^{-1}$ in excellent agreement with our theoretical results of 359.0 and $354.0 \mathrm{~cm}^{-1}$, respectively. Two isotopic shifts of -25.4 and $-9.8 \mathrm{~cm}^{-1}$ were observed for the split $3^{1} 4^{1}\left(e^{\prime}\right)$ levels. From the wave function analysis we find two states (levels 20 and 21 in Tab. 5) clearly correlating to this transition with computed isotope shifts of -30.2 and $-20.1 \mathrm{~cm}^{-1}$, respectively. Both levels correspond to $a_{1}$ symmetry and show shifts in reasonable though not great agreement with one of the shifts 
determined experimentally. It is clear that only one of the two states can correlate to the $3^{1} 4^{1}\left(e^{\prime}\right)$ transition in ${ }^{14} \mathrm{~N}^{16} \mathrm{O}_{3}$. The only $b_{1}$ state computed nearby should be the other component and its isotopic shift of $-8.8 \mathrm{~cm}^{-1}$ would be in rather good agreement with the experimental value. These isotopic shift together with the shifts obtained for ${ }^{15} \mathrm{~N}^{16} \mathrm{O}_{3}$ yield additional support for the assignment of the prominent $1492 \mathrm{~cm}^{-1}$ feature to the $3^{1} 4^{1}$ combination band. The remaining results cannot be compared to experimental results and thus may serve as a prediction for possible future experimental investigations.

\section{Conclusions}

In the present work a new full-dimensional diabatic PES model for the $\tilde{X}, \tilde{A}$, and $\tilde{B}$ state of the $\mathrm{NO}_{3}$ radical is presented and the vibrational dynamics of the $\tilde{X}$ state is investigated using this model. The new PES model accurately represents a large number (roughly 90000 energies) of high-level MRCI $a b$ initio data, though still leaving room for improvement. Using the same technology, an accurate adiabatic dipole surface for the $\tilde{X}$ state was determined also. The new model is used in the present study to compute the IR spectrum of $\mathrm{NO}_{3}$ up to approximately $2000 \mathrm{~cm}^{-1}$. In addition, isotopic shifts are determined for the two species ${ }^{15} \mathrm{~N}^{16} \mathrm{O}_{3}$ and ${ }^{14} \mathrm{~N}^{18} \mathrm{O}^{16} \mathrm{O}_{2}$, which yields important additional data to settle a long lasting debate about the assignment of a prominent spectral feature observed experimentally at $1492 \mathrm{~cm}^{-1}$.

Vibrational frequencies are computed in good to excellent agreement with experimental values for most of the computed and measured levels. The observed errors are smaller for the fundamentals than for most of the combination bands and the largest error is observed for the disputed level at $1492 \mathrm{~cm}^{-1}$ for which the present calculations yield $1438 \mathrm{~cm}^{-1}$. Since the analytical representation of the PES data is very accurate with rms errors of only $100 \mathrm{~cm}^{-1}$ up to $1 \mathrm{eV}$ above minimum, the deficiencies in the PES are most likely due to the electronic structure calculations. However, effects of the data point sampling cannot be ruled out and will be investigated in forthcoming work. All computed frequencies are lower than the experimental values indicating that the MRCI method slightly underestimates the binding strength in $\mathrm{NO}_{3}$. This might be due to the very complicated electronic structure in which the mixing to the Jahn-Teller active $\tilde{B}$ state plays a significant role, which also leads to complications in the nuclear dynamics.

The computed vibrational eigenstates are also analysed in terms of the harmonic oscillator basis and diabatic electronic states. It is found that the $\tilde{B}$ state contributes between $2-5 \%$ to the vibrational states associated with the $\tilde{X}$ state while the contributions of the $\tilde{A}$ state are negligible. The analysis of the eigenstates in terms of vibrational quanta is possible for most transitions of lower energy though the significant anharmonicity and strong mode mixing present in $\mathrm{NO}_{3}$ renders this progressively meaningless for higher transitions. Nevertheless, most assignments based on the present calculations are in agreement with experimental analyses from high-resolution IR spectra. This is particularly important for the assignment of the debated $1492 \mathrm{~cm}^{-1}$ feature. According to our calculations this is clearly a $3^{1} 4^{1}$ combination band in agreement with previous high-level theoretical studies. The $\nu_{3}$ fundamental is predicted around $1022 \mathrm{~cm}^{-1}$, 
also in agreement with all other theoretical results. The evidence for this assignment is further strengthened by the computed isotopic shifts for the ${ }^{15} \mathrm{~N}^{16} \mathrm{O}_{3}$ isotopologue. For all levels for which the assignments are undisputed the computed and experimentally determined isotope shifts are within $2 \mathrm{~cm}^{-1}$, usually much better. This is also true for the $3^{1} 4^{1}$ transition which has a significantly larger isotope shift than the $3^{1}$ transition. If the $1492 \mathrm{~cm}^{-1}$ feature was assigned to the $\nu_{3}$ fundamental, the isotope shift would be clearly inconsistent with the theoretical prediction. One striking disagreement we find between the present calculations and experimental observations of the IR intensities. The present calculations using an accurate representation of the adiabatic dipole surfaces for the $\tilde{X}$ state predict a fairly strong absorption for the $3^{1}$ transition but this feature is at most very weak in experimental IR spectra. It is likely that the absorption intensities are influenced by vibronic coupling to the $\tilde{B}$ state and a much more elaborate diabatic model for the dipole operator would be needed to account for these effects. This will be subject to future work because it is beyond the scope of the present study.

The present results show that the developed diabatic PES model yields very good results for the $\tilde{X}$ state and thus similarly accurate results can be expected for the nuclear dynamics in the excited states. The results also give further support for the assignment of the $1492 \mathrm{~cm}^{-1}$ feature to a $3^{1} 4^{1}$ combination band rather than the $\nu_{3}$ fundamental, which is expected around $1022 \mathrm{~cm}^{-1}$. This work will be continued by further improvements of the diabatic PES model which will be used for forthcoming quantum dynamics studies of this interesting but challenging molecular system. 


\section{Acknowledgments}

We are grateful for generous financial support by the Deutsche Forschungsgemeinschaft (DFG). We thank

Uwe Manthe for many helpful and enlightening discussions on quantum dynamics and numerical algorithms.

[1] P. Hautefeuille and J. Chappuis, C. R. Acad. Sci. Paris 92, 80 (1881).

[2] G. Sprenger, Z. Elektrochem. 37, 674 (1931).

[3] R. P. Wayne, Chemistry of Atmospheres (Oxford University Press, Oxford, 2000), 3rd ed.

[4] R. P. Wayne, I. Barnes, P. Biggs, J. P. Burrows, C. E. Canosamas, J. Hjorth, G. Lebras, G. K. Moortgat, D. Perner, G. Poulet, et al., Atmos. Environ., Part A 25, 1 (1991).

[5] E. J. Jones and O. R. Wulf, J. Chem. Phys. 5, 873 (1937).

[6] G. Schott and N. Davidson, J. Am. Chem. Soc. 80, 1841 (1958).

[7] H. H. Nelson, L. Pasternack, and J. R. McDonald, J. Chem. Phys. 79, 4279 (1983).

[8] H. F. Davis, P. I. Ionov, S. I. Ionov, and C. Wittig, Chem. Phys. Lett. 215, 214 (1993).

[9] L. Valachovic, C. Riehn, K. Mikhaylichenko, and C. Wittig, Chem. Phys. Lett. 258, 644 (1996).

[10] K. Mikhaylichenko, C. Riehn, L. Valachovic, A. Sanov, and C. Wittig, J. Chem. Phys. 105, 6807 (1996).

[11] H. S. Johnston, H. F. Davis, and Y. T. Lee, J. Phys. Chem. 100, 4713 (1996).

[12] A. Weaver, D. W. Arnold, S. E. Bradforth, and D. M. Neumark, J. Chem. Phys. 94, 1740 (1991).

[13] A. Deev, J. Sommar, and M. Okumura, J. Chem. Phys. 122, 224305 (2005).

[14] M. Okumura, J. Stanton, A. Deev, and J. Sommar, Phys. Scripta 73, C64 (2006).

[15] K. Takematsu, N. C. Eddingsaas, D. J. Robichaud, and M. Okumura, Chem. Phys. Lett. 555, 57 (2013).

[16] T. Codd, M.-W. Chen, M. Roudjane, J. F. Stanton, and T. A. Miller, J. Chem. Phys. 142, 184305 (2015).

[17] M. E. Jacox and W. E. Thompson, J. Phys. Chem. A 114, 4712 (2010).

[18] H. H. Nelson, L. Pasternack, and J. R. McDonald, J. Phys. Chem. 87, 1286 (1983).

[19] T. Ishiwata, I. Fujiwara, Y. Naruge, K. Obi, and I. Tanaka, J. Phys. Chem. 87, 1349 (1983).

[20] K. Kawaguchi, E. Hirota, T. Ishiwata, and I. Tanaka, J. Chem. Phys. 93, 951 (1990).

[21] K. Kawaguchi, T. Ishiwata, I. Tanaka, and E. Hirota, Chem. Phys. Lett. 180, 436 (1991).

[22] T. Ishiwata, I. Tanaka, K. Kawaguchi, and E. Hirota, J. Mol. Spectrosc. 153, 167 (1992).

[23] K. Kawaguchi, T. Ishiwata, E. Hirota, and I. Tanaka, Chem. Phys. 231, 193 (1998).

[24] T. Ishiwata, Y. Nakano, K. Kawaguchi, E. Hirota, and I. Tanaka, J. Phys. Chem. A 114, 980 (2010).

[25] K. Kawaguchi, N. Shimizu, R. Fujimori, J. Tang, T. Ishiwata, and I. Tanaka, J. Mol. Spectrosc. 268, 85 (2011).

[26] R. Fujimori, N. Shimizu, J. Tang, T. Ishiwata, and K. Kawaguchi, J. Mol. Spectrosc. 283, 10 (2013).

[27] K. Kawaguchi, R. Fujimori, J. Tang, and T. Ishiwata, J. Phys. Chem. A 117, 13732 (2013).

[28] K. Kawaguchi, R. Fujimori, J. Tang, and T. Ishiwata, J. Mol. Spectrosc. 314, 73 (2015).

[29] R. R. Friedl and S. P. Sander, J. Phys. Chem. 91, 2721 (1987).

[30] B. Kim, P. L. Hunter, and H. S. Johnston, J. Chem. Phys. 96, 4057 (1992).

[31] D. Forney, W. E. Thompson, and M. E. Jacox, J. Chem. Phys. 99, 7393 (1993).

[32] M. E. Jacox and W. E. Thompson, J. Chem. Phys. 129 (2008).

[33] H. Beckers, H. Willner, and M. E. Jacox, Chem. Phys. Chem. 10, 706 (2009).

[34] J. F. Stanton, J. Chem. Phys. 126, 134309 (2007).

[35] A. Lund and K. Thuomas, Chem. Phys. Lett. 44, 569 (1976).

[36] J. F. Olsen and L. Burnelle, J. Am. Chem. Soc. 92, 3659 (1970).

[37] N. C. Baird and K. F. Taylor, Chem. Phys. Lett. 80, 83 (1981).

[38] R. D. Davy and H. F. Schaefer III, J. Chem. Phys. 91, 4410 (1989).

[39] B. Kim, B. L. Hammond, W. A. Lester, Jr., and H. S. Johnston, Chem. Phys. Lett. 168, 131 (1990).

[40] V. R. Morris, S. Bhatia, and J. H. Hall, Jr., J. Phys. Chem. 94, 7414 (1990).

[41] J. F. Stanton, J. Gauss, and R. J. Bartlett, J. Chem. Phys. 94, 4084 (1991).

[42] J. F. Stanton, J. Gauss, and R. J. Bartlett, J. Chem. Phys. 97, 5554 (1992).

[43] U. Kaldor, Chem. Phys. Lett. 166, 599 (1990).

[44] U. Kaldor, Chem. Phys. Lett. 185, 131 (1991).

[45] A. Stirling, I. Pápai, J. Mink, and D. R. Salahub, J. Chem. Phys. 100, 2910 (1994).

[46] W. Eisfeld and K. Morokuma, J. Chem. Phys. 113, 5587 (2000).

[47] W. Eisfeld and K. Morokuma, J. Chem. Phys. 114, 9430 (2001).

[48] R. A. Ogg, Jr., J. Chem. Phys. 21, 2079 (1953).

[49] W. A. Guillory and H. S. Johnston, J. Chem. Phys. 42, 2457 (1965).

[50] S. C. Bhatia and J. H. Hall, Jr., J. Phys. Chem. 84, 3255 (1980).

[51] V. R. Morris, S. C. Bhatia, and J. H. Hall, Jr., J. Phys. Chem. 91, 3359 (1987).

[52] J. E. D. Morris and H. S. Johnston, J. Chem. Phys. 47, 4282 (1967).

[53] W. Eisfeld and K. Morokuma, J. Chem. Phys. 119, 4682 (2003).

[54] M. Mayer, L. S. Cederbaum, and H. Köppel, J. Chem. Phys. 100, 899 (1994)

[55] S. Mahapatra, W. Eisfeld, and H. Köppel, Chem. Phys. Lett. 441, 7 (2007).

[56] S. Faraji, H. Köppel, W. Eisfeld, and S. Mahapatra, Chem. Phys. 347, 110 (2008).

[57] J. F. Stanton, Mol. Phys. 107, 1059 (2009).

[58] J. F. Stanton and M. Okumura, Phys. Chem. Chem. Phys. 11, 4742 (2009). 
[59] C. S. Simmons, T. Ichino, and J. F. Stanton, J. Phys. Chem. Lett. 3, 1946 (2012).

[60] Z. Homayoon and J. M. Bowman, J. Chem. Phys. 141, 161104 (2014).

[61] W. Eisfeld, O. Vieuxmaire, and A. Viel, J. Chem. Phys. 140, 224109 (2014).

[62] W. Eisfeld and A. Viel, J. Chem. Phys. 146, 034303 (2017).

[63] W. Domcke, D. R. Yarkony, and H. Köppel, eds., Conical Intersections: Electronic Structure, Dynamics and Spectroscopy (World Scientific, Singapore, 2004).

[64] H. C. Longuet-Higgins, Adv. Spectrosc. 2, 429 (1961).

[65] W. Lichten, Phys. Rev. 131, 229 (1963).

[66] W. Lichten, Phys. Rev. 164, 131 (1967).

[67] F. T. Smith, Phys. Rev. 179, 111 (1969).

[68] M. Baer, Chem. Phys. 15, 49 (1976).

[69] C. A. Mead and D. G. Truhlar, J. Chem. Phys. 77, 6090 (1982).

[70] C. A. Mead, J. Chem. Phys. 78, 807 (1983).

[71] T. Pacher, L. S. Cederbaum, and H. Köppel, J. Chem. Phys. 89, 7367 (1988).

[72] T. Pacher, C. A. Mead, L. S. Cederbaum, and H. Köppel, J. Chem. Phys. 91, 7057 (1989).

[73] T. Pacher, H. Köppel, and L. S. Cederbaum, J. Chem. Phys. 95, 6668 (1991).

[74] T. Pacher, L. S. Cederbaum, and H. Köppel, Adv. Chem. Phys. 84, 293 (1993).

[75] H. Köppel, W. Domcke, and L. S. Cederbaum, Adv. Chem. Phys. 57, 59 (1984).

[76] R. Cimiraglia, J. P. Malrieu, M. Persico, and F. Spiegelmann, J. Phys. B-At. Mol. Opt. 18, 3073 (1985).

[77] W. Domcke and C. Woywod, Chem. Phys. Lett. 216, 362 (1993).

[78] G. J. Atchity and K. Ruedenberg, Theo. Chem. Acc. 97, 47 (1997).

[79] H. Nakamura and D. G. Truhlar, J. Chem. Phys. 115, 10353 (2001).

[80] H. Nakamura and D. G. Truhlar, J. Chem. Phys. 117, 5576 (2002).

[81] H. Nakamura and D. G. Truhlar, J. Chem. Phys. 118, 6816 (2003).

[82] M. S. Schuurman and D. R. Yarkony, J. Chem. Phys. 127, 094104 (2007).

[83] B. N. Papas, M. S. Schuurman, and D. R. Yarkony, J. Chem. Phys. 129, 124104 (2008).

[84] X. Zhu and D. R. Yarkony, J. Chem. Phys. 130, 234108 (2009).

[85] X. Zhu and D. R. Yarkony, J. Chem. Phys. 132, 104101 (2010).

[86] X. Zhu and D. R. Yarkony, J. Chem. Phys. 136, 174110 (2012).

[87] H. Ndome, R. Welsch, and W. Eisfeld, J. Chem. Phys. 136, 034103 (2012).

[88] H. Ndome and W. Eisfeld, J. Chem. Phys. 137, 064101 (2012).

[89] N. Wittenbrink, H. Ndome, and W. Eisfeld, J. Phys. Chem. A 117, 7408 (2013).

[90] F. Venghaus and W. Eisfeld, J. Chem. Phys. 144, 114110 (2016).

[91] A. J. C. Varandas, F. B. Brown, C. A. Mead, D. G. Truhlar, and N. C. Blais, J. Chem. Phys. 86, 6258 (1987).

[92] H.-J. Werner, B. Follmeg, and M. H. Alexander, J. Chem. Phys. 89, 3139 (1988).

[93] H. Werner and W. Meyer, J. Chem. Phys. 74, 5802 (1981).

[94] G. Hirsch, R. J. Buenker, and C. Petrongolo, Mol. Phys. 70, 835 (1990).

[95] C. R. Evenhuis and M. A. Collins, J. Chem. Phys. 121, 2515 (2004).

[96] C. R. Evenhuis, X. Lin, D. H. Zhang, D. Yarkony, and M. A. Collins, J. Chem. Phys. 123, 134110 (2005).

[97] O. Godsi, C. R. Evenhuis, and M. A. Collins, J. Chem. Phys. 125, 104105 (2006).

[98] X. Zhu, J. Y. Ma, D. R. Yarkony, and H. Guo, J. Chem. Phys. 136, 234301 (2012).

[99] V. C. Mota and A. J. C. Varandas, J. Phys. Chem. A 112, 3768 (2008).

[100] A. Viel and W. Eisfeld, J. Chem. Phys. 120, 4603 (2004).

[101] W. Eisfeld and A. Viel, J. Chem. Phys. 122, 204317 (2005).

[102] A. Viel, W. Eisfeld, S. Neumann, W. Domcke, and U. Manthe, J. Chem. Phys. 124, 214306 (2006).

[103] A. Viel, W. Eisfeld, C. R. Evenhuis, and U. Manthe, Chem. Phys. 347, 331 (2008).

[104] A. V. Marenich and J. E. Boggs, J. Chem. Phys. 122, 024308 (2005).

[105] A. V. Marenich and J. E. Boggs, Chem. Phys. Lett. 404, 351 (2005).

[106] D. Opalka and W. Domcke, Chem. Phys. Lett. 494, 134 (2010).

[107] D. Opalka and W. Domcke, J. Chem. Phys. 132, 154108 (2010).

[108] X. Zhu and D. R. Yarkony, J. Chem. Phys. 140, 024112 (2014).

[109] W. Eisfeld, J. Chem. Phys. 134, 054303 (2011).

[110] H. D. Meyer, U. Manthe, and L. S. Cederbaum, Chem. Phys. Lett. 165, 73 (1990).

[111] U. Manthe, H. D. Meyer, and L. S. Cederbaum, J. Chem. Phys. 97, 3199 (1992).

[112] U. Mante, J. Phys.: Condens. Matter 29, 253001 (2017).

[113] U. Manthe, J. Chem. Phys. 128, 064108 (2008).

[114] C. Evenhuis, G. Nyman, and U. Manthe, J. Chem. Phys. 127, 144302 (2007).

[115] R. Wodraszka, J. Palma, and U. Manthe, J. Phys. Chem. A 116, 11249 (2012).

[116] U. Manthe, J. Chem. Phys. 105, 6989 (1996).

[117] J. C. Light and T. Carrington, Adv. Chem. Phys. 114, 263 (2000).

[118] K. Kawaguchi, T. Narahara, R. Fujimori, J. Tang, and T. Ishiwata, J. Mol. Spectrosc. 334, 10 (2017).

[119] E. Hirota, J. Mol. Spectrosc. 310, 99 (2015).

[120] K. Tada, W. Kashihara, M. Baba, T. Ishiwata, E. Hirota, and S. Kasahara, J. Chem. Phys. 141, 184307 (2014). 